\title{
Validation of the prolapse quality-of-life questionnaire (P-QOL): An Afrikaans version in a South African population
}

\author{
C Brandt, ${ }^{1} \mathrm{PhD}$ (Physiotherapy); C van Rooyen, ${ }^{2} \mathrm{M}$ Comm; H S Cronjé, ${ }^{3} \mathrm{MB} \mathrm{ChB}, \mathrm{MD}$
}

${ }^{1}$ Department of Physiotherapy, University of the Free State, Bloemfontein, South Africa

${ }^{2}$ Department of Biostatistics, University of the Free State, Bloemfontein, South Africa

${ }^{3}$ Private practice, Centurion, Pretoria, South Africa

Corresponding author: C Brandt (gnftcb@ufs.ac.za)

Background. The prolapse quality-of-life questionnaire (P-QOL) has been validated and translated into eight languages. The lack of an Afrikaans version of the P-QOL limits studies in Afrikaans-speaking patients with pelvic organ prolapse (POP).

Objective. To validate an Afrikaans version of the P-QOL in a South African population.

Methods. The P-QOL was translated into Afrikaans by a medical translator and three gynaecologists. This descriptive study determined construct validity comparing 25 symptomatic $(64.1 \%, n=39)$ and 14 asymptomatic $(35.9 \%, n=39)$ participants' median domain scores. The POP stage was determined according to the POP quantification (POP-Q) scale and compared with their domain scores by means of percentages. A second P-QOL was completed and the stability determined by the test-retest method. The Cronbach alpha was used to determine internal consistency and the kappa value to determine measure of agreement.

Results. Symptomatic participants had higher median domain scores than asymptomatic participants. All asymptomatic participants had stage 0 POP and $33.3 \%$ of symptomatic participants had stage III POP. Stability was good, with an average of above $50 \%$. The mean Cronbach alpha value was 0.94 and the kappa value indicated moderate to good strength of agreement between items $(\mathrm{K}=0.41-0.80)$.

Conclusion. The Afrikaans P-QOL was found to be valid and reliable to determine quality of life in women with POP, correlating with the findings of other validation studies and supporting the evidence that the P-QOL is a high-quality disease-specific quality-of-life questionnaire.

S Afr J Obstet Gynaecol 2016;22(2):38-41. DOI:10.7196/SAJOG.2016.v22i2.1077

Pelvic organ prolapse (POP) occurs in $46-73 \%$ of women in South Africa (SA) and may also be associated with other pelvic floor dysfunctions. ${ }^{[1]}$ The assessment of quality of life (QOL) is becoming increasingly important in determining the outcome of pelvic floor reconstructive surgery, as well as other pelvic floor disorders. ${ }^{[2]}$

POP is mostly benign, but it is distressing and disabling, with a large effect on the patient's QOL. ${ }^{[3]}$ The multifactorial pathophysiology may be the cause of associated symptoms such as bladder, bowel, sexual, and even pain symptoms. ${ }^{[4]}$ The symptoms may lead to physical, social, psychological, domestic, and/or sexual limitations in the patient's activities of daily living. ${ }^{[5]}$ A survey done by Muller ${ }^{[6]}$ in the USA found that women with POP experienced compromised bladder and bowel control as most limiting their QOL. Ranked second was the inability to enjoy physical activities such as sport. They also emphasised satisfaction with conservative and surgical management as an important factor in determining QOL. ${ }^{[6]}$ It is important to validly and reliably determine the QOL from the patient's perspective, because it has been indicated that the validity of QOL outcomes based on physicians' perspectives should be interpreted with caution. ${ }^{[7]}$ Changes in QOL from the patient's perspective should therefore be seriously considered when treatment and treatment outcomes of POP are determined. ${ }^{[8]}$

The prolapse quality-of-life questionnaire (P-QOL) is one of only a few validated and reliable condition-specific questionnaires developed to assess the impact of urogenital prolapse on the QOL of patients. The questionnaire covers various domains of life which include general health, prolapse impact, role limitations, physical limitations, social limitations, personal relationships, emotional problems, sleep/energy disturbances and prolapse severity. ${ }^{[9]}$

The P-QOL has been successfully translated into eight languages, and includes versions in Italian, ${ }^{[5]}$ Turkish, ${ }^{[10]}$ German, ${ }^{[1]]}$ Portuguese, ${ }^{[12]}$ Dutch, ${ }^{[2]}$ Thai, ${ }^{[8]}$ and most recently Persian. ${ }^{[13]}$ The lack of a validated Afrikaans questionnaire investigating QOL in patients experiencing prolapse limits studies and effective outcome measurement in Afrikaans-speaking patients in SA. ${ }^{[15]}$ A second problem is that the P-QOL was originally developed for a European population; this might raise the question as to its validity in a multicultural SA population.

The purpose of the study was therefore to validate the P-QOL in an Afrikaans-speaking SA population.

\section{Methods}

The observational, descriptive study was approved by the Ethics Committee of the Faculty of Health Sciences of the University of the Free State, Bloemfontein, SA. Written consent and permission were obtained from the participants and institutions where the study was conducted.

\section{Translation of the P-QOL}

The original English P-QOL questionnaire was translated into Afrikaans by an independent medical translator. The translated Afrikaans version was reviewed and translated back into English by 
three independent urogynaecologists. The urogynaecologists agreed that the original content had been retained and that no ambiguity was present. No changes were made in the translated version.

\section{Pilot study}

The translation was followed by a pilot study $(n=5)$ to confirm the readability and participants comprehension of the questions. Participants were asked to complete the questionnaire and were then interviewed by the researchers. No problems were identified and no changes were made to the translated Afrikaans version of the P-QOL. The same methodological procedures were followed as described for the main study in the following sections.

\section{Sampling}

A convenience sample was used, consisting of 40 women meeting the inclusion criteria (Table 1). The eligibility criteria were aligned with the validated versions of the P-QOL, and applied to the demographics of a SA population.

\section{Procedures}

The eligible participants had to complete an informed consent document, a P-QOL and a demographic data form after the study procedures had been explained to them by the researchers. The same urogynaecologist determined the POP-Q score for all participants according to clinical and ultrasonography findings. The completed questionnaires and forms were checked by the researchers to ensure that all the information was gathered.

Following consultation, each participant was given a second blank P-QOL in an addressed envelope, to complete and mail back after 2 weeks in order to determine the stability of the questionnaire by a test-retest analysis. The date on which the questionnaire had to be completed was indicated by a note on the envelope. Reminders were sent to all participants to complete the second questionnaire.

\section{Data analysis}

The SAS software package (SAS, USA) and Excel (Version 2010) (Microsoft, USA) software were used for statistical analysis. The two questionnaires' construct validity was determined by assessing the domain score of symptomatic and asymptomatic participants, and then comparing it with the POP-Q score to determine criterion validity by means of percentages. The test-retest method was used to indicate stability of the P-QOL, the kappa value to calculate the measure of agreement, and the Cronbach alpha to measure the internal consistency.

Descriptive statistics were used to explain the demographic data. Medians and percentiles were calculated for continuous data, and frequencies and percentages were calculated to describe categorical data.

\section{Results}

A total of 40 women were enrolled into the study. Twenty-five women $(64.1 \%, n=39)$ were symptomatic and 14 women $(35.9 \%$,

Table 1. Eligibility criteria
Inclusion criteria
Women attending private practices and provincial outpatient clinics
Women literate in Afrikaans
Exclusion criteria
Women $<18$ years
Women $>90$ years
Current pregnancy
Childbirth or pelvic surgery in the past 6 months
Active urinary tract infections
Cognitive impairment

Table 2. Summary of demographic data of participants

\begin{tabular}{lll}
\hline & Symptomatic $(\boldsymbol{n = 2 5})$ & Asymptomatic $(\boldsymbol{n = 1 4})$ \\
\hline Age (years), median & 60 & 45.5 \\
Body mass (kg), median & 67.5 & 80 \\
Parity, $n$ & & $(n=12)^{*}$ \\
0 & 0 & 0 participants \\
1 & 1 & 3 participants \\
2 & 7 & 4 participants \\
$\geq 3$ & 17 & 5 participants \\
Method of delivery, $n$ & & $(n=12)^{*}$ \\
NVD & 20 & 9 participants \\
Caesarean section & 1 & 2 participants \\
Both & 4 & 1 participant \\
POP-Q findings, $n / N$ (\%) & & 14 (35.9) \\
Stage 0 & 0 & 0 \\
Stage I & $4(10.3)$ & 0 \\
Stage II & $7(18.0)$ & 0 \\
Stage III & $13(33.33)$ & 0 \\
Stage IV & $1(2.6)$ & \\
NVD = normal vaginal delivery.. & \\
${ }^{*}$ Two asymptomatic participants did not complete the parity question (including 'Method of delivery') & &
\end{tabular}


RESEARCH

Table 3. P-QOL domain scores of symptomatic and asymptomatic participants

\begin{tabular}{lllll}
\hline & \multicolumn{2}{c}{ Symptomatic (median) } & \multicolumn{2}{c}{ Asymptomatic (median) } \\
\cline { 2 - 5 } Prolapse QOL domain & Assessment 1 score $(\boldsymbol{n})$ & Assessment 2 score $(\boldsymbol{n})$ & Assessment 1 score $(\boldsymbol{n})$ & Assessment 2 score $(\boldsymbol{n})$ \\
\hline General health perceptions & $25(25)$ & $25(18)$ & $25(14)$ & $25(7)$ \\
Prolapse impact & $33.33(25)$ & $33.33(18)$ & $33.33(14)$ & $66.67(7)$ \\
Role limitations & $33.33(25)$ & $33.33(18)$ & $0(14)$ & $0(7)$ \\
Physical limitations & $25(24)$ & $33.33(18)$ & $0(13)$ & $33.33(7)$ \\
Social limitations & $22.22(13)$ & $22.22(12)$ & $0(9)$ & $22.22(4)$ \\
Personal relationships & $16.67(13)$ & $33.33(11)$ & $0(10)$ & $0(3)$ \\
Emotions & $22.22(25)$ & $16.67(18)$ & $16.67(14)$ & $0(7)$ \\
Sleep/energy & $50(25)$ & $33.33(18)$ & $12.5(14)$ & $0(7)$ \\
Severity measures & $16.67(24)$ & $16.67(18)$ & & $0(7)$
\end{tabular}

$n=39)$ were asymptomatic, with one data form incomplete with regard to the POP-Q score. Table 2 shows a summary of the demographic and clinical characteristics of symptomatic and asymptomatic participants.

Construct validity was determined by assessing the domain score of symptomatic and asymptomatic participants, and then comparing it with the POP-Q findings to determine criterion validity. The P-QOL domain scores of symptomatic participants were mostly higher compared with asymptomatic participants, indicating a poorer QOL (Table 3). Fourteen (35.9\%) asymptomatic participants were classified as stage 0 on the POP-Q system and $64.1 \%$ of the symptomatic participants $(n=25)$ had POP, ranging from stage II to IV. All asymptomatic participants $(100 \%, n=14)$ were stage 0 on the POP-Q system and the majority of the symptomatic participants $(52.0 \%, n=13 / 25)$ were at stage III.

Stability was measured by means of the test-retest method to determine the consistency of the responses by participants (response rate $65 \%, n=40$. All the reliability percentages were above $50 \%$ (Fig. 1). According to the interpretation of Birkimer and Brown, ${ }^{[15]}$ the median percentage of $69.2 \%$, the sample size of 40 , and the number of disagreements equalling $<3$ indicate a non-chance agreement.

The measure of agreement between specific questions was determined by means of calculating the kappa value (Fig. 2). The lowest kappa value was 0.12 for question 38, indicating poor strength in agreement. The highest kappa value was 0.65 for question 29, indicating good strength in agreement ( $\kappa$ range $0.61-0.80$ ). The strength of agreement was fair in 4 questions ( $\kappa$ range $0.21-0.40$ ), moderate in 11 ( $\kappa$ range $0.41-0.60$ ) and good in 2 ( $\kappa$ range 0.61 0.80 ). The majority of questions, 13 of 20 , therefore had a moderate to good strength of agreement.

The internal consistency was determined by means of the Cronbach alpha. A Cronbach alpha $>0.7$ is interpreted as acceptable. Table 4 shows that assessments 1 and 2 had a mean Cronbach alpha score of 0.94 , indicating very high reliability.

\section{Discussion}

Approximately half of the population in SA is female,,$^{[14]}$ and POP may occur in up to three-quarters of them. ${ }^{[1]}$ Pelvic floor disorders have been described as mainly being a QOL disorder ${ }^{[16]}$ Subjective improvement and improvement in QOL are main goals of management of patients with POP (pre- and/or postoperatively) and need to be clinically evaluated by means of a valid outcome measure. ${ }^{[6,16]}$
Table 4. Internal consistency (Cronbach $\alpha$ coefficient) for P-QOL domains between assessment 1 and 2

\begin{tabular}{lll}
\hline & \multicolumn{2}{c}{ Cronbach $\boldsymbol{\alpha}$} \\
\cline { 2 - 3 } $\begin{array}{l}\text { Prolapse QOL } \\
\text { domain }\end{array}$ & $\begin{array}{l}\text { Assessment 1 } \\
\text { score }\end{array}$ & $\begin{array}{l}\text { Assessment 2 } \\
\text { score }\end{array}$ \\
\hline General health perceptions & $*$ & $*$ \\
Prolapse impact & $*$ & $*$ \\
Role limitations & 0.89 & 0.88 \\
Physical limitations & 0.90 & 0.76 \\
Social limitations & 0.64 & 0.67 \\
Personal relationships & 0.61 & 0.40 \\
Emotions & 0.91 & 0.91 \\
Sleep/energy & 0.83 & 0.58 \\
Severity measures & 0.36 & 0.46 \\
Mean score & 0.94 & 0.94 \\
*The domains 'general health perceptions' and 'prolapse impact' had only one item and the \\
internal consistency could not be calculated.
\end{tabular}

This study determined validity and reliability aspects of an Afrikaans version of the $\mathrm{P}-\mathrm{QOL}$ in a province in SA where Afrikaans is widely spoken. Content validity was determined in a similar way as described by previous validation studies of the P-QOL. ${ }^{[5]}$ The content was found to be valid after review by a medical translator, panel of experts and pilot study.

The construct validity was indicated by the median domain scores of the symptomatic patients being higher than the asymptomatic patients' scores, except for 'general health perceptions' and 'prolapse impact' scores. The results correlate with the findings of other validation studies which indicated statistical significant differences between the scores of symptomatic and asymptomatic patients. Lenz et al. ${ }^{[11]}$ and De Oliveira et al. ${ }^{[12]}$ also found the domain scores of 'general health perceptions' to be similar for both groups, because of the fact that this category consists of only one question and can be affected by symptoms or diseases not related to POP. A finding significant to this study was the lack in differences in scores relating to 'prolapse impact'. The difference in the sample size for which each of these medians was calculated may affect the interpretation of these results. Another possibility might be that POP is multifactorial and can include an interaction and coexistence of several pelvic floor disorders affecting the experience of symptoms in even the minor ('asymptomatic') stages of $\mathrm{POP}^{[2]}$ 


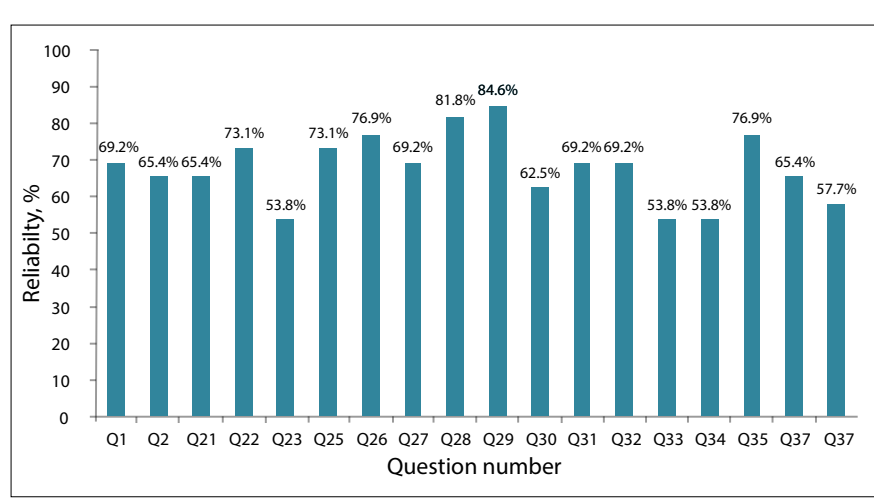

Fig. 1. Test-retest reliability of the nine P-QOL domains. $\left({ }^{\star}\right.$ Test retest reliability could not be calculated for questions 24 and 36.)

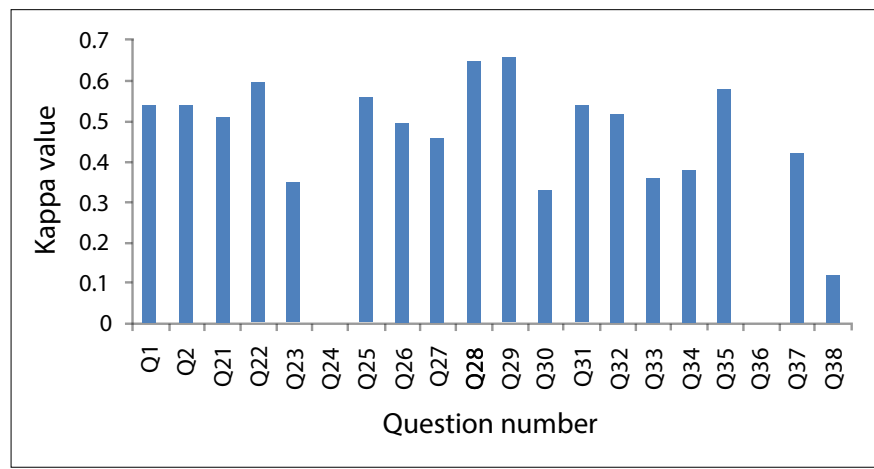

Fig. 2. Kappa values indicating strength of agreement. $\left({ }^{\star}\right.$ Kappa values could not be calculated for questions 24 and 36.)

Lower domain scores also showed a relationship with less severe stages of POP, as assessed by the POP-Q system, indicating good criterion validity similar to the findings of previous studies.

The methods used in this study to calculate construct and criterion validity differed from most other studies which used the MannWhitney $U$ test and the Spearman's rho correlation, respectively. This difference in methodology limits specific comparison of some of the validity findings of this study with those of the previous studies, though the conclusive findings were similar. The use of different statistical methods, all finding the same results, can however be seen as a strength to substantiate the findings from different validation studies.

Reliability measures correlate with previous findings on translated versions of the P-QOL. ${ }^{[2,5,8,10,12,13]}$ Stability measures in this study indicated a non-chance agreement with the test-retest method, while the kappa value indicated moderate to good strength of agreement between questions, for the majority of the questions. A very high internal consistency was indicated by a mean Cronbach alpha of 0.94 during the first and second assessments.

Unfortunately the response rate for the second assessment was low as a result of poor patient compliance, even though patients were reminded about completing and sending back their questionnaires. Despite this limitation, a statistical analysis was still possible for this relatively small population from which the sample was drawn.
The limited population also raised some concern as to the interpretation of the content validity found in this study. It must be taken into consideration that the descent of the Afrikaans-speaking participants included in this study may differ from the descent of Afrikaans-speaking patients in other demographical areas in SA. Clinical and cultural differences may affect the validity of an Afrikaans questionnaire, and it is therefore recommended that it should be tested in different regions in SA.

\section{Conclusion}

This study found the translated Afrikaans version of the P-QOL to have good content, construct and criterion validity, as well as very high stability, strength of agreement and internal consistency. This correlates with the validity and reliability of other translated versions of the P-QOL, supporting the evidence that the P-QOL is a high-quality disease-specific QOL questionnaire. It can be recommended that the P-QOL be translated into other African languages, and especially to determine content validity in the different African cultures.

Acknowledgements. Departments of Urogynaecology and Biostatistics of the University of the Free State, L Taute, Y Sutherland, P Ackerman, H Luckhoff, F Dry and C van Dyk for their field work.

Author contributions. CB was involved with protocol, project development, data collection and management, and manuscript writing, HSC with protocol, project development, and manuscript writing, and CvR with data analysis. The Afrikaans version of the P-QOL can be obtained from the corresponding author.

\section{References}

1. Cronjé HS. Pelvic Organ Prolapse. Bloemfontein: University of the Free State. http://www. topicsinmedicine.org/pelvic\%20organ\%20prolapse.html (accessed 16 April 2011).

2. Claerhout F, Moons P, Ghesquiere S, Verguts J, de Ridder D, Deprest J. Validity, reliability and responsiveness of a Dutch version of the prolapse quality-of-life (P-QoL) questionnaire. Int Urogynecol J 2012;21:569-578. http://dx.doi.org/10.1007/s00192-009-1081-9

3. Cronjé HS. Pelvic organ prolapse. In: Kruger TF, Botha MH, eds. Clinical Gynaecology, 4th ed. Cape Town: Juta \& Co., 2011:487-515,

4. Fritel X, Varnoux N, Zins M, Breart G, Ringa V. Symptomatic pelvic organ prolapse at midlife, quality of life, and risk factors. Obstet Gynecol 2009;113(3):609-616. http://dx.doi.org/10.1097/ quality of life, and risk

5. Digesu GA, Santamato S, Khullar V, et al. Validation of an Italian version of the prolapse quality of life questionnaire. Eur J Obstet Gynecol Reprod Biol 2003;106(2):184-192.

6. Muller N. Pelvic organ prolapse: A patient-centred perspective on what women encounter seeking diagnosis and treatment. Aust N Z Cont J 2010;16(3):70-80.

7. Srikrishna S, Robinson D, Cardozo L, Gonzalez J. Is there a difference in patient and physician quality of life evaluation in pelvic organ prolapse? Int Urogynecol J 2008;19:517-520. http://dx.doi quality of life evaluation in pelvic or

8. Manchana T, Bunyavejchevin S. Validation of the prolapse quality of life (P-QOL) questionnaire in Thai version. Int Urogynecol J 2010;21:985-993. http://dx.doi.org/10.1007/s00192-010-1107-x

9. Digesu GA, Khullar V, Cardozo L, Robinson D, Salvatore S. P-QOL: A validated questionnair to assess the symptoms and quality of life of women with urogenital prolapse. Int Urogynecol J 2005;16:176-181. http://dx.doi.org/10.1007/s00192-004-1225-x

10. Cam C, Sakalli M, Ay P, Aran T, Cam M, Karateke A. Validation of the prolapse quality of life questionnaire (P-QOL) in a Turkish population. Eur J Obstet Gynecol Reprod Biol 2007;135(1):132135. http://dx.doi.org/10.1016/j.ejogrb.2007.06.009

11. Lenz H, Stammer H, Brocker K, Rak M, Scherg H, Sohn C. Validation of a German version of the P-QOL questionnaire. Int Urogynecol J 2009;20:641-649. http://dx.doi.org/10.1007/s00192-009-0809-x

12. De Oliveira MS, Tamanini JTN, de Aguiar Cavalcanti G. Validation of the prolapse quality-of-life questionnaire (P-QoL) in Portuguese version in Brazilian woman. Int Urogynecol J 2009;20:11911202. http://dx.doi.org/10.1007/s00192-009-0934-6

13. Nojomi M, Digesu GA, Khullar V, et al. Validation of Persian version of the prolapse quality of life questionnaire (P-QOL). Int Urogynecol J 2012;23:229-233. http://dx.doi.org/10.1007/s00192-011-1529-6

14. Statistics South Africa. http://beta2.statssa.gov.za/?page_id=593 (accessed 27 February 2015).

15. Birkimer JC, Brown JH. Back to basics: Percentage agreement measures are adequate, but there are easier ways. J Appl Behav Anal 1979;12:535-543.

16. Lowenstein L, FitzGerald MP, Kenton K, et al. Patient-selected goals: The fourth dimension in assessment of pelvic floor disorders. Int Urogynecol J Pelvic Floor Dysfunct 2008;19(1):81-84. 